\title{
Review of the Available Scientific Evidence on Multiple Chemical Sensitivity Syndrome
}

\author{
Alberto Frutos Pérez-Surio ${ }^{1}$, Gema Sancho Monllor ${ }^{2}$, Ignacio Frutos Pérez-Surio ${ }^{3}$
}

1. Department of Preventive Medicine and Public Health. University of Zaragoza. Zaragoza, Spain

2. Expert in Documentation and Information Systems. Zaragoza Region Government. Zaragoza, Spain

3. Environmental Chemistry Specialist Technician. Nilsson Laboratories. Zaragoza, Spain

\begin{abstract}
Background: Multiple Chemical Sensitivity (MCS) Syndrome poses many faces and it is related to previous exposure to substances found in the environment, at well-tolerated concentration by the general population. There are a lot of sources of exposure and there is no typical clinical pattern, as well as several degrees of severity in the subjects affected by MCS Syndrome. For all these reasons, the MCS Syndrome becomes a complex disease with difficult diagnosis and management. The aim of this study is to review and to update the available scientific evidence on MCS Syndrome. Methodology: A systematic revision of the available evidence has been made, and updated the available evidence. The following sources were used: Medline, Embase, PSYCINFO, Toxline, UpToDate, IBSST, CISDOC, The Cochrane Library and Centre for Reviews and Dissemination (CRD). Two reviewers independently made the selection of recovered articles, applying the inclusion and exclusion criteria for the final selection of studies. There were identified 613 studies of which 27 were selected for the 5 analyzed clinical areas: 1) Epidemiology, 2) Clinical manifestations and co-morbid clinical conditions, 3) Diagnosis of MCS syndrome, 4) Physical, Psychological and Social impact on the Quality of Life 5) Therapeutic and prevention approaches for MCS Syndrome. Results: The estimation of MCS's prevalence can range between $0.02 \%$ and $0.04 \%$, increasing to $19 \%$ in people with an added diagnosis of allergy. The most frequently affected systems in the MCS Syndrome are the digestive tract, the cardiovascular one and the skin. Also, the mental sphere is affected with certain frequency. The MCS's diagnosis can be difficult, due to the variety of symptoms the disease can show from the beginning and because of the wide range of different definitions about the same disease that can be made. The clinical suspicion after a correct anamnesis and physical exam and the use of the Quick Environmental Exposure and Sensitivity Inventory (QEESI), in Spanish version can help with the diagnosis. People affected by MCS Syndrome seems to decrease their quality of life in a significant way, even reaching self-isolation in an attempt to reduce the exposition to trigger substances or due to the serious physical deterioration in case of continuous expositions. The more effective therapeutic intervention consists on avoiding new re-exposure to the leading substances. Conclusions: The MCS is frequently associated with a previous allergic subject. It can affect multiple systems simultaneously, most often the gastrointestinal and cardiovascular systems, the skin and the mental sphere. The non-specific symptoms and the lack of consensus about the definition of MCS deter an early diagnosis of the disease. There is a Spanish version of the QEESI of reference. It is believed that the adoption of measures to sensitize the general population about MCS, could influence the reduction of exposures to trigger substances and improve understanding of this disease and people who suffer it. Actually, the best preventive measure is to avoid exposure to the triggering substances.
\end{abstract}

Keywords: Multiple chemical sensitivity; systematic review; low-dose toxicity; risk assessment

\section{INTRODUCTION}

Multiple Chemical Sensitivity (MCS) is described as a complex condition that involves a set of symptoms attributed to exposure to extremely low levels of a wide variety of environmental chemical $^{1}$. It involves the exposure to potentially harmful chemical, physical, or biological agents in the environment or to environmental factors that may include ionizing radiation, pathogenic organisms, or toxic chemicals ${ }^{2}$. The symptoms experienced by many individuals get them, in some cases, exhaust ${ }^{3,4}$.

The pathogenic mechanisms involved in MCS are not clearly established ${ }^{5-7}$. There are several biologically plausible hypotheses that warrant further scientific research to explain the underlying 
mechanism or modes of action of $\mathrm{MCS}^{8}$. In addition, methods of diagnosis and treatment have not yet been agreed upon by the medical profession ${ }^{9,10}$.

Given the uncertainty regarding the mechanisms, criteria for diagnosis and treatment methods of $\mathrm{MCS}^{11,12}$ there is the need to review new research on MCS to improve the understanding, prevention and treatment of MCS. However, these calls for further investigations have determined priority areas for scientific and research community in the field of health and the environment. Elucidate the biological basis of $\mathrm{MCS}^{13}$ provide guidelines for clinical diagnosis and improve treatment option ${ }^{14}$. It must also identify the natural history and the true prevalence of this condition ${ }^{15}$.

The aim of this review is to update the available evidence in the scientific literature.

\section{Methodology}

A systematic revision of the available evidence has been made. The search was done by consulting the following databases: Medline, Embase, The Cochrane Library and the Centre for Reviews and Dissemination (which include the Database of Abstracts of Reviews of Effects, the National Health Service Economic Evaluation and the Health Technology Assessment Database). We have also searched on specialized databases in this field: PsycINFO, Pubpsych, Toxline, OSH, Update and CISDOC. A description of the search strategies is available as supplementary material (upload file Annex 1). The publication date was set between 2010 and 2015, as a Spanish previous review was done in 2010. Two reviewers independently made the selection of recovered articles, applying to the inclusion and exclusion criteria for the definitive selection of studies. Inclusion criteria: Population based case control studies with results of prevalence or incidence of MCS; Original research publications with MCS patients in whom the effectiveness of intervention with one or more drugs or other therapeutic measures is studied; Studies that describe MCS symptoms; Qualitative studies that address physical, psychological and social impact, and quality of life; Narrative and editorial reviews that provide a novel approach or new working hypothesis in the research of MCS. Study exclusion criteria: Papers on the study of electromagnetic radiation; Population with different primary diagnosis of MCS; Studies with different main problem of MCS; Studies dealing MCS but do not respond to the area of interest. Due to heterogeneity of the included studies no statistical analysis was performed (meta-analysis or quantitative synthesis).

\section{RESUlts}

There were identified 613 studies of which 27 were selected (figure 1 describes the PRISMA flow diagram) for the 5 analyzed clinical areas: 1) Epidemiology, 2) Clinical manifestations and co-morbid clinical conditions, 3) Diagnosis of MCS syndrome, 4) Physical, Psychological and Social impact on the Quality of Life 5) Therapeutic and prevention approaches for MCS Syndrome. Extensive description of included studies (Table 1) and reasons for excluded studies (Table 2) are also reported.

1. Epidemiology: In the observational study done by Lee et al. $2013^{16}$ a prevalence of $19.1 \%$ of MCS in allergic population is estimated and established risk factors change of address and use household cleaning products. The study of Nogué et al. $2011^{17}$ estimated prevalence in the Spanish population between $0.02 \%$ and $0.04 \%$.

2. Clinical manifestations and comorbidity: Caccamo et al. $2013^{18}$ indicate that patients affected by MCS appear most frequently gastrointestinal and cardiovascular disorders in relation to suspected cases of MCS. In cases of suspected MCS it is most often headache-neurological, respiratory, skin, musculoskeletal and immunological symptoms in diagnosed cases of MCS. 30\% of patients have comorbidities, highlighting main remaining comorbidities in chronic fatigue syndrome and fibromyalgia $70 \%$. In Holst et al. $2011 \mathrm{a}^{19}$ erythema intensity induced by capsaicin in MCS patients with eczema and the outbreak area in patients with symptoms induced by odorous chemicals studied. The symptoms studied included in upper airway and downs, CNS, allergic rhinitis, asthma, eczema and food allergy. They performed prick tests (skin tests) to airborne allergens, analyzing the intensity of erythema and measuring the response of the skin neurovascular spectroscopy using polarized light. In the study of Holst et al. $2011 \mathrm{~b}^{20}$ reactivity to pain, hyperalgesia, temporal summation effect and neurogenic inflammation in patients with MCS is evaluated. The clinical trial of Berg et al. $2011^{21}$ investigates the relationship between skin reactions to various chemicals, and the sensitivity reported by the patient from inhalation of airborne chemicals. Patch tests are performed. Allergic skin responses (RAC) and non-allergic skin responses (NRAC) had statistically significant positive 
association with more severe groups compared to the group that does not mind any of the exposures included in the questionnaire on MCS. Individuals relate chemical sensitivity not show an increase in allergic reactions. Katerndahl et al. $2012^{22}$ evaluate the prevalence of chemical intolerance, comorbidities and psychiatric disorders in patients with Primary care setting. They are linear relationship between the number of mental disorders and the prevalence of chemical intolerance, stressing that primary care centers, chemical intolerance, is often unrecognized, still prevalent in lowincome population, and frequently presented comorbidity with a large class medical and psychiatric conditions, having to make an active search for cases. They also highlight that psychiatric comorbidities contribute to functional limitations and increased use of health care. Skovbjerg et al. $2012^{23,24}$ investigate the association between depressive symptoms and four domains related to idiopathic environmental intolerance (IEI), determining whether the association could be confused by the low social support and major life events. Significant correlations are moderate to high scales for symptoms of central nervous system (CNSS); mucosal symptoms (MUSS); consequences for social activities (CSAS); and chemical hypersensitivity (CHS). In addition, the features of personal perception and somatosensory amplification and regional perceptions are associated with IEI. It failed to show association between repressive coping style and IEI, either with alexithymia (inability to identify and verbalize the emotions), but with some properties of it, such as negative emotional reactions, defensiveness and difficulty identifying feelings. The cross-sectional study by Nordin et al. $2013^{25}$ investigates whether sensitivity to environmental noise is related to the perception of stress and sensitivity to environmental odors, supporting the hypothesis that noise sensitivity is associated with perceived stress and sensitivity to smell.

3. Diagnosis of MCS: Through a cross-sectional study, Skovbjerg et al. $2012^{26}$ evaluated questionnaire translation QEESI applied Danish population, verifying the reliability and validity, and sensitivity and specificity. The authors propose to investigate in the future the combined use of the scales "Chemical intolerance" and "impact on activities of daily living", because it is a shorter, but equally valid alternative to the QEESI complete questionnaire. Barnig et al. $2013^{27}$ hypothesis review the pathophysiology of MCS and found no differences between healthy and sick people, or medical identification or cognitive development or serum cortisol. Martini et al. $2013^{28}$ made an overview of MCS specifying the critical points of the case definition and diagnosis in relation to the workplace. They propose a diagnostic protocol for suspected MCS, including a first phase with detailed interview using several questionnaires, blood and urine analysis, the signs and indications of the patient and spirometry. A second phase is indicated when suspected MCS and no other diseases or other modified aspects exist. In these cases, the second phase consists of the following tests: psychological evaluation, neurophysiological, allergy testing, genetic polymorphisms, determine different chemicals, metals and other metabolites in biological samples, as well as research on the metabolism and the use of detoxifying agents. In the cross-sectional study of Mena et al. $2013^{29}$ translated into Spanish the quick quiz exposure and environmental sensitivity (QEESI) and adapted to the Spanish population. The univariate analysis for the description of the population is made by comparing the median scores between diagnosed and undiagnosed MCS subjects, and assessing the internal consistency of the scales.

4. Physical, psychological and social impact. Quality of Life: Gibson et al. $2011^{30}$ conducted a qualitative study to investigate the impact of MCS in their lives. Respondents refer social and occupational isolation; need to take many precautions; they indicate that a cultural effort is needed to create safe environments (work, cinema, transport, etc.) where you can interact with others. Moreover, refer incomprehension, ignorance about their sickness, loneliness, guilty fellness by high demand for maintaining relationships. It is mentioned that a solution may be to educate others about MCS, although not everyone shares this approach. They note that Internet and telephone help maintain social relations in the context of isolation caused by the disease. Finally, they report feeling outside world, as mere observers. In another qualitative study, Soderholm et al. $2011^{31}$ intended to clarify how individuals experience living with sensory hyperreactivity (HRS), the impact on accessibility, economic security and social relations. According to the authors, the HRS is a form of intolerance to odors, including MCS and IEI. Difficulties are taking a shuttle, visit public buildings and facilities, and indicate that finding a suitable place to live is almost impossible. With regard to economic security: reduction of income due to the difficulty of living, increased expenses due to HRS, lack of support from the authorities, and difficulties to manage their finances. Finally, with regard to social relations, refer to: socializing has become difficult and traumatic, among other conditions. The study 
of Dupas et al. $2013^{32}$ presents the social and labor implications for people with MCS, even losing the job.

5. MCS Therapeutic Approach: In 2013 Genuis $^{33}$ presented as currently therapeutic alternatives, the use of desensitizing immunotherapy; Profile unfavorable risk / benefit of steroids and immunosuppressive drugs for the treatment of this syndrome; the lack of success of cognitive therapy and other commercial alternatives (Dynamic Neural Retraining System ${ }^{\mathrm{TM}}$ ) and; eliminating xenobiotics by physiological processes (the toxicokinetics phases of metabolism or biotransformation, and excretion) or exogenous interventions, that appear to decrease detoxification immune disorder, and improve clinical status. It indicated that physiological treatments are superior and sustainable compared to psychological therapies and highlights the role of Public Health and the work of health education. The study of Ralph et al. $2011^{34}$, regarding a case of a woman with MCS for permethrin, emphasizes environmental research as a tool to make the right treatment and prevention measures. In the same line Waddick $2011^{35}$ highlighted, in a case report, the importance of lifestyle with avoidance of exposure through sustainable urban development. In the United States, Mischley et al. $2013^{36}$ describe the results of the use of intranasal reduced glutathione (inGSH), highlighting the efficacy and safety of inGSH for respiratory and central nervous system diseases.

With a preventive strategy, a descriptive study was found, regarding a case of a woman diagnosed with MCS, investigating the clinical changes in relation to changes in exposure and re-exhibition to accidental contaminants. Environmental toxicological research specifically identified as causal origin of MCS clinic patient, accidental exposure to permethrin insecticide biocide properties, so that preventive measures are in line to avoid exposure to this chemical.

\section{DISCUSSION AND CONCLUSION}

In 1996 a WHO/IPCS Workshop has suggested to use as an appropriate descriptor of MCS the broader term "Idiopathic Environmental Intolerances (IEI)", in order to incorporate "a number of disorders sharing similar symptomatologies", and research was strongly encouraged ${ }^{37}$. MCS is an acquired disorder characterized by recurrent symptoms, referable to multiple organ systems, occurring in response to demonstrable exposure to many chemically unrelated compounds at doses below those established in the general population to cause harmful effects ${ }^{38}$.

Over $15 \%$ of the general population has mechanisms excessive response to certain chemicals or environmental stimuli and 5\% of cases are pathological and exceed the adaptive capacity of the organism; MCS is an acquired disease, characterized by progressive loss of tolerance to the presence in the environment of various chemical agents ${ }^{39}$ such as household cleaning products, colognes, perfumes, solvents or hydrocarbons ${ }^{40}$. In the middle of last century the first cases of these patients became ill when exposed to substances well below harmful levels to health were reported.

Sensitivity to chemicals is a toxicological concept ${ }^{41}$, contained in the dose-response relationship ${ }^{42}$. Sensitivity also includes the concept of hypersensitivity ${ }^{43}$, although controversy surrounds the nature of effects from very low exposures ${ }^{44}$. Research into the possible mechanisms of MCS is far from complete $^{45}$.

Its etiology and pathophysiology remain a mystery, although several inconclusive theories are postulated. Therefore, the diagnosis is based on symptoms presented by patients, as there is no laboratory test blood or urine, and no specific complementary examination in order to confirm the diagnosis ${ }^{46-49}$. The symptoms differ among individuals who have to change their lifestyle to cope with the disease $\mathrm{e}^{50,51}$.

A clear predominance of involvement in females $(80 \%)$ and the most common chemicals for developing symptoms are household cleaning products and fragrances, toiletries and cosmetics is observed. That is why it has made out a literature review of the MCS and the search for evidence that would link these two variables; there has not been published ${ }^{9,52}$.

Fragrances and other odorants could, however, be associated with symptoms as claimed by MCS symptomatics ${ }^{53,54}$, because they are recognizable stimuli, but fragrance has not been demonstrated to be causal in the usual sense ${ }^{55}$.

Scientific Committee on Consumer Safety recently published (June 2012) an opinion on fragrance allergens in cosmetic products ${ }^{56}$. Fukuyama et al. used long-term sensitization followed by low-dose 
challenge to evaluate sensitization by well-known Th2 type sensitizers [trimellitic anhydride (TMA) and toluene diisocyanate (TDI)] and a Th1 type sensitizer [2,4-dinitrochlorobenzene (DNCB)]. This long-term sensitization method would be useful for detecting environmental chemical-related hypersensitivity ${ }^{57}$.

Even today, MCS is not part of the ICD-10 (International Classification of Diseases) although associated with various diseases ${ }^{58}$ and pose a disability in activities of daily living of sufferers ${ }^{59}$ and for workers ${ }^{60-62}$.

In conclusion, we can say that the uncertainty in the etiological attribution, not being clear causal origin of MCS is maintained. The update also notes that there is no clear diagnosis. More studies are presented to indicate the impact on quality of life. No specific measures found treatment except symptomatic measures. In the area of prevention, it is designated as primary preventive measure avoidance of exposure and re-exposure.

\section{TABLES}

Table 1. Summary of evidence tables of included articles

\section{Epidemiology}

\begin{tabular}{|c|c|c|c|c|c|}
\hline REFERENCE & $\begin{array}{l}\text { STUDY / } \\
\text { POPULATION }\end{array}$ & INTERVENTION & RESULTS & CONCLUSIONS & COMMENTS \\
\hline $\begin{array}{l}\text { Lee } \text { et al. } \\
2013^{15} \text {, South } \\
\text { Korea }\end{array}$ & $\begin{array}{l}\text { Aims } \\
\text { To evaluate the } \\
\text { prevalence and related } \\
\text { factors of MCS in } \\
\text { patients with allergic } \\
\text { diseases. } \\
\text { Design } \\
\text { Observational study. } \\
\text { Period of execution } \\
\text { Not indicated. } \\
\text { Population } \\
196 \text { patients with } \\
\text { allergic diseases. }\end{array}$ & $\begin{array}{l}\text { They classify patients } \\
\text { according to scores on } \\
\text { the questionnaire } \\
\text { QEESI: } \\
\text { Group 1: very } \\
\text { suggestive of suffering } \\
\text { MCS (symptoms } \\
\text { severity score } \geq 40 \text { and } \\
\geq 40 \text { chemical } \\
\text { intolerance) } \\
\text { Group 2: little } \\
\text { suggestive of suffering } \\
\text { MCS (lower scores) } \\
\text { Statistic analysis } \\
\text { Subsequently, a } \\
\text { univariate analysis was } \\
\text { performed. }\end{array}$ & $\begin{array}{l}\text { Prevalence of: } \\
\text { - Asthma: } 39.4 \%(\mathrm{n}=77) \\
\text { - Allergic rhinitis: } 70.4 \%(\mathrm{n}=138) \\
\text { - Atopic dermatitis: } 30.1 \%(\mathrm{n}=59) \\
34 \text { patients were included in Group } 1 . \\
\text { MCS prevalence of } 19.1 \% . \\
\text { Demographic characteristics and } \\
\text { patterns of allergic disease were not } \\
\text { different between groups. } \\
\text { MCS is related to change of address, } \\
\text { adjusted OR [95\% CI } 5.29(1.39, \\
\text { 20.09)] and the use of household } \\
\text { cleaning products more than once a } \\
\text { week, adjusted OR [5.20 95\% CI } \\
(1.19,22.86)] .\end{array}$ & $\begin{array}{l}\text { The risk of MCS in allergic } \\
\text { patients is higher in patients } \\
\text { who have changed address or } \\
\text { frequently used household } \\
\text { cleaning products. } \\
\text { In this study, although MCS } \\
\text { is not dependent allergic } \\
\text { diseases, both are } \\
\text { environmental diseases may } \\
\text { be related. } \\
\text { Further studies are needed to } \\
\text { establish relationships } \\
\text { between MCS and allergic } \\
\text { diseases. }\end{array}$ & $\begin{array}{l}\text { Information on the origin of } \\
\text { the sample selection, } \\
\text { variable definitions (change } \\
\text { of address, use household } \\
\text { cleaning products), and MCS } \\
\text { prevalence of allergic } \\
\text { diseases such failure. }\end{array}$ \\
\hline
\end{tabular}

\section{Clinical manifestations and comorbidity}

\begin{tabular}{|c|c|c|c|c|c|c|}
\hline REFERENCE & STUDY & POPULATION & $\begin{array}{l}\text { INTERVENTION } \\
/ \\
\text { COMPARISON }\end{array}$ & CLINIC / COMORBIDITY & CONCLUSIONS & COMMENTS \\
\hline $\begin{array}{l}\text { Caccamo et al. } \\
2013^{18} \text {, Italy }\end{array}$ & \begin{tabular}{|l} 
Objetive \\
Compare the \\
distribution of \\
genetic \\
polymorphism of \\
cytochrome \\
P450 and Aryl \\
hydrocarbon \\
receptor \\
(xenobiotic \\
sensor) in 3 \\
cohorts. \\
Study design \\
Case-control. \\
Period of \\
realization \\
Not specified.
\end{tabular} & $\begin{array}{l}\text { Inclusion criteria } \\
\text { People from different Italian } \\
\text { regions, with full or partial } \\
\text { diagnosed with MCS } \\
\text { hypersensitivity. } \\
\text { Groups } \\
\text { MCS Group: } 156 \text { patients were } \\
\text { diagnosed by Cullen and QEESI } 20 \\
\text { to } 30 \text { points. } \\
\text { Middle Ages (EM): } 49 \text { (11) } \\
\text { SMCs Group: } 94 \text { (79M / 15H); } \\
\text { QEESI 10-20 points. } \\
\text { MS: } 49 \text { years (12) }\end{array}$ & $\begin{array}{l}\text { Comparative } \\
\text { description of } \\
\text { comorbidities } \\
\text { registered in the } \\
\text { group of MCS and } \\
\text { SMCS in the group }\end{array}$ & $\begin{array}{l}\text { HCS Group (approximate figures } \\
* \text { ) } \\
\approx 50 \% \text { neurological-headache } \\
\approx 50 \% \text { Respiratory } \\
\text { Musculoskeletal } \approx 42 \% \\
\approx 37 \% \text { immunological } \\
\text { Gastrointestinal } \approx 33 \% \\
\text { Cardiovascular } \approx 30 \% \\
\text { Skin } \approx 29 \% \\
\approx 30 \% \text { without comorbidities } \\
\text { SMCS Group (approximate } \\
\text { figures } * \text { ) } \\
\approx 58 \% \text { neurological-headache } \\
\approx 62 \% \text { Respiratory } \\
\text { Musculoskeletal } \approx 42 \% \\
\approx 40 \% \text { immunological } \\
\text { Gastrointestinal } \approx 26 \% \\
\text { Cardiovascular } \approx 16 \% \\
\text { Skin } \approx 39 \% \\
\approx 6 \% \text { without comorbidities }\end{array}$ & $\begin{array}{l}\text { DOES NOT } \\
\text { APPLY }\end{array}$ & $\begin{array}{l}\text { The study does not } \\
\text { aims to analyze } \\
\text { differences between } \\
\text { groups comorbidities } \\
\text { Only about } 30 \% \text { of } \\
\text { people diagnosed } \\
\text { with MCS have no } \\
\text { associated } \\
\text { comorbidities, but } \\
\text { this figure is reduced } \\
\text { to approximately } 6 \% \\
\text { in the case of people } \\
\text { with suspected MCS. } \\
\text { Clinical data } \\
\text { extracted from a } \\
\text { chart }\end{array}$ \\
\hline
\end{tabular}


Alberto Frutos Pérez-Surio et al.

\begin{tabular}{|c|c|c|c|c|c|c|}
\hline REFERENCE & STUDY & POPULATION & $\begin{array}{l}\text { INTERVENTION / } \\
\text { COMPARISON }\end{array}$ & CLINIC / COMORBIDITY & CONCLUSIONS & COMMENTS \\
\hline $\begin{array}{l}\text { Berg et al. } \\
2011^{33}, \\
\text { Denmark }\end{array}$ & $\begin{array}{l}\text { Objetive } \\
\text { Researching } \\
\text { general } \\
\text { population the } \\
\text { relationship } \\
\text { between skin } \\
\text { reactions to } \\
\text { various } \\
\text { chemicals, and } \\
\text { the sensitivity } \\
\text { reported by the } \\
\text { patient to the } \\
\text { inhalation of } \\
\text { airborne } \\
\text { chemicals } \\
\text { itself. } \\
\text { Study design } \\
\text { Clinical trial. } \\
\text { Period of } \\
\text { realization } \\
\text { June 2006-June } \\
2008\end{array}$ & $\begin{array}{l}\text { Recruitment } \\
\text { From 7,931 } \\
\text { people in the } \\
\text { civil registry. } \\
\text { Copenhagen } \\
\text { area. } \\
\text { Including } 3,471 \\
(43.8 \%) . \\
\text { age range } \\
\text { between } 18 \text { and } \\
69 \text { a. } \\
\text { Invitation to } \\
\text { participate in a } \\
\text { general health } \\
\text { checkup (Health } \\
\text { 2006) }\end{array}$ & $\begin{array}{l}\text { Intervention } \\
\text { Patch test: the answer } \\
\text { classifies as ( } 3,460 \\
\text { people): } \\
\text { Allergic skin response } \\
\text { (RCA): redness and } \\
\text { dermis infiltration } \\
\text { No allergic skin response } \\
\text { (RCNA): irritative } \\
\text { response, follicular, or } \\
\text { doubtful. } \\
\text { Prick test: (2,232 people) } \\
\text { Positive: wheal diameter } \\
\geq 3 \text { mm } \\
\text { 4 groups according } \\
\text { consequences (severity) } \\
\text { attributed to inhalation of } \\
\text { chemicals in airborne } \\
\text { Group 1: Undisturbed } \\
\text { exhibitions included in } \\
\text { the questionnaire. } \\
\text { Group 2: exposure- } \\
\text { related symptoms but no } \\
\text { impact on daily life. } \\
\text { Group 3: refers } \\
\text { adjustments in lifestyles. } \\
\text { Group 4: refers } \\
\text { adjustments in social or } \\
\text { work life. }\end{array}$ & $\begin{array}{l}\text { Patch test: } \\
\text { RCA (univariate analysis): } \\
\text { A> severity of symptoms (groups } 1 \text { to 4), } \\
\text { RCA. Statistically significant (p <0.05) for } \\
\text { groups } 3 \text { and } 4 \text { with respect REFERENCE } \\
\text { (group 1) } \\
\text { Group 3: } \\
\text { OR 1,37 (IC95\%: } 1,04 \text { a 1,81) } \\
\text { Group 4: } \\
\text { OR 2,03 (IC95\%: } 1,10 \text { a 3,74) } \\
\text { RCA (Multivariate analysis } * \text { ): } \\
\text { There is no association between the severity of } \\
\text { symptoms and RCA. No statistical significance } \\
\text { (p>0.05) } \\
\text { RCNA: } \\
\text { RCNA (univariate analysis): } \\
\text { A> severity of symptoms (groups } 1 \text { to 4),> } \\
\text { RCA. Statistically significant (p <0.05) for } \\
\text { groups } 3 \text { and } 4 \text { with respect REFERENCE } \\
\text { (group 1) } \\
\text { Group 3: } \\
\text { OR 1,55 (IC95\%: } 1,15 \text { a 2,08) } \\
\text { Group 4: } \\
\text { OR 2,83 (IC95\%: } 1,55 \text { a 5,15) } \\
\text { RCNA (Multivariate analysis *): } \\
\text { Association seen with> severity of symptoms } \\
\text { (group 4) and }>\text { RCNA. } \\
\text { Group 4: } \\
\text { OR 2,63 (IC95\%: } 1,39 \text { a 5,01) p=0,003 } \\
\text { No association between severity of symptoms } \\
\text { and RCN to the other groups (p> } 0.05 \text { ) were } \\
\text { observed } \\
\text { Prick Test: } \\
\text { RESULTS no association between test and } \\
\text { severity of symptoms (groups) is identified. } \\
\text { RESULTS NOT included in multivariate } \\
\text { analysis and not shown }\end{array}$ & $\begin{array}{l}\text { People who refers } \\
\text { chemical } \\
\text { sensitivity, show } \\
\text { an increase in non- } \\
\text { allergic skin } \\
\text { reactions, basing } \\
\text { this information on } \\
\text { reading the patch } \\
\text { test } 48 \text { hours. }\end{array}$ & $\begin{array}{l}\text { Possible } \\
\text { limitations } \\
\text { described by } \\
\text { authors due to } \\
\text { reading the patch } \\
\text { test } 48 \text { hours } \\
\text { rather than } 72 \\
\text { hours. } \\
\text { *: RCA and } \\
\text { RCNA, sex, age, } \\
\text { eczema, atopic } \\
\text { dermatitis, } \\
\text { asthma, } \\
\text { depression, } \\
\text { anxiety, smoking, } \\
\text { social status and } \\
\text { educational level: } \\
\text { more complete } \\
\text { information } \\
\text { model includes } \\
\text { the following } \\
\text { variables is } \\
\text { described } \\
\text { adjustment. }\end{array}$ \\
\hline
\end{tabular}

\begin{tabular}{|c|c|c|c|c|c|}
\hline REFERENCE & $\begin{array}{l}\text { STUDY } \\
\end{array}$ & POPULATION & $\begin{array}{l}\text { INTERVENTION / } \\
\text { COMPARISON }\end{array}$ & CLINIC / COMORBIDITY & $\begin{array}{l}\text { CONCLUSIONS / } \\
\text { COMMENTS }\end{array}$ \\
\hline $\begin{array}{l}\text { Katerndahl et } \\
\text { al. } 2012^{34} \text {, U.S. }\end{array}$ & $\begin{array}{l}\text { Objetive } \\
\text { Assess medical } \\
\text { and psychiatric } \\
\text { comorbidities } \\
\text { in a community } \\
\text { of people with } \\
\text { or without } \\
\text { MCS } \\
\text { Study design } \\
\text { Transversal } \\
\text { study. } \\
\text { Period of } \\
\text { realization } \\
\text { Not indicate }\end{array}$ & $\begin{array}{l}\text { Recruitment } \\
2 \text { primary care centers: } \\
\text { Centre A: Hispanic } \\
\text { population with low } \\
\text { incomes } \\
\text { Center B: Hispanic } \\
\text { middle class population } \\
\text { and non-Hispanic } \\
\text { Sample } \\
400 \text { patients } \geq 18 \text { years } \\
\text { of age: } \\
\text { Activity A: } 270 \text { (68\%) } \\
\text { Center B: } 130 \text { (32\%) } \\
\text { Reason query: no } \\
\text { acute conditions } \\
\text { Characteristics } \\
\text { Average age: } 47.4 \text { years } \\
14.7 \\
148 \text { (37\%) men and } 252 \\
\text { (63\%) women } \\
\text { Groups }\end{array}$ & $\begin{array}{l}\text { Intervenyion } \\
\text { All questionnaires filled: } \\
\text { sociodemographic characteristics } \\
\text { Quick Environmental Exposure and } \\
\text { Sensitivity Inventoriy (QEESI). } \\
\text { MCS define whether score } \geq 40 \text { for } \\
\text { scale chemical intolerance scale } \\
\text { and severity of symptoms. } \\
\text { Apply without discrimination, } \\
\text { intolerance and chemical MCS } \\
\text { Primary Care Evaluation of Mental } \\
\text { Disorders (PRIME-MD). Detection } \\
\text { of psychiatric disorders in the } \\
\text { previous month. } \\
\text { Statistic analysis } \\
\text { Descriptive } \\
\text { asthma, allergies, autism, multiple } \\
\text { sclerosis, arthritis, diabetes, gastro- } \\
\text { intestinal, mood disorders, chemical } \\
\text { intolerance, systemic erythemic } \\
\text { lupus: refer family and personal }\end{array}$ & $\begin{array}{l}\text { RESULTS shown only statistically significant } \\
\text { differences } \\
\text { G1 vs. G2: (personal record): } \\
\text { Allergies: } 43 \text { (53\%) vs. } 129 \text { (40\%) } \\
\text { Mood altered state: } 44 \text { (54\%) vs. } 64 \text { (20\%) } \\
\text { chemical intolerance: } 19 \text { (24\%) vs. } 24 \text { (8\%) } \\
\text { G1 vs. G2: (family history): } \\
\text { Gastro-intestinal disorders: } 21 \text { (26\%) vs. } 31 \\
\text { (10\%) } \\
\text { Mood altered state: } 31 \text { (38\%) vs. } 56 \text { (18\%) } \\
\text { chemical intolerance: } 16 \text { (20\%) vs. } 24 \text { (8\%) } \\
\text { Systemic Lupus Erythematosus } 7 \text { (9\%) vs. } 9 \\
\text { (3\%) } \\
\text { G1 vs. G2: (mental clinic): } \\
\text { Major depression: } 69 \text { (85\%) vs. } 106(33 \%) \\
\text { Generalized Anxiety: } 63 \text { (78\%) vs. } 67 \text { (21\%) } \\
\text { Panic attacks: } 44 \text { (54\%) vs. } 53 \text { (17\%) } \\
\text { Alcohol: } 30,37 \%) \text { vs. } 63 \text { (20\%) } \\
\text { Somatization disorders: } 74 \text { (91\%) vs. } 218 \\
\text { (68\%) } \\
\text { Relationship mental health and chemical }\end{array}$ & $\begin{array}{l}1 \text { in } 5 \text { people who use } \\
\text { health services Primary } \\
\text { Care (US) for non- } \\
\text { acute conditions, } \\
\text { presents MCS. } \\
\text { Mental disorders are } \\
\text { more common in } \\
\text { people with MCS and } \\
\text { allergies and other } \\
\text { mood disorders. } \\
\text { The gastro-intestinal, } \\
\text { mood disorders, lupus } \\
\text { chemical intolerance } \\
\text { and family history are } \\
\text { most often mentioned } \\
\text { by people with MCS } \\
\text { COMMENTS } \\
\text { Autoquestionaries for } \\
\text { information } \\
\text { There is a pre-selection } \\
\text { of those who come for }\end{array}$ \\
\hline
\end{tabular}


Review of the Available Scientific Evidence on Multiple Chemical Sensitivity Syndrome

\begin{tabular}{|c|c|c|c|c|c|}
\hline & & $\begin{array}{l}\text { MCS (G1): } 81(20.3 \%) \\
\text { people } \\
\text { Without MCS (G2): } \\
319(79.7 \%) \text { people }\end{array}$ & $\begin{array}{l}\text { history is explored. } \\
\text { major depression, generalized } \\
\text { anxiety, panic attacks, alcohol abuse } \\
\text { and somatization disorders. }\end{array}$ & $\begin{array}{l}\text { intolerance: } \\
\text { A> prevalence of MCS,> number of possible } \\
\text { mental disorders }\end{array}$ & $\begin{array}{l}\text { non-acute conditions } \\
\text { Participation in the } \\
\text { study is paid (\$5) }\end{array}$ \\
\hline
\end{tabular}

\begin{tabular}{|c|c|c|c|c|c|}
\hline REFERENCE & STUDY & POPULATION & $\begin{array}{l}\text { INTERVENTION / } \\
\text { COMPARISON }\end{array}$ & CLINIC / COMORBIDITY & CONCLUSIONS \\
\hline $\begin{array}{l}\text { Skovbjerg et al. } \\
2012 \mathrm{a}^{35} \text {, } \\
\text { Denmark }\end{array}$ & $\begin{array}{l}\text { Objetive } \\
\text { To analyze the } \\
\text { association between } \\
\text { psychological } \\
\text { disorders and } \\
\text { idiopathic } \\
\text { environmental } \\
\text { intolerance * (IEI) } \\
\text { and determine } \\
\text { whether social } \\
\text { support and major } \\
\text { life events could be } \\
\text { confounding factors } \\
\text { Study design } \\
\text { Transversal study } \\
\text { Period of } \\
\text { realization } \\
\text { They do not tell. } \\
\text { *: IEI disorder } \\
\text { characterized by } \\
\text { nonspecific } \\
\text { symptoms of several } \\
\text { attributed by the } \\
\text { person to exposure } \\
\text { to common } \\
\text { chemicals in the air } \\
\text { organs. }\end{array}$ & $\begin{array}{l}\text { Recruitment } \\
\text { 1,024 people invited } \\
\text { to participate. } \\
\text { Group 1 (G1): } \\
\text { general population. } \\
787 \text { people } \\
\text { Group } 2 \text { (G2): } 237 \\
\text { people. Danish } \\
\text { Research Center for } \\
\text { symptoms common } \\
\text { aerosols and diagnosis } \\
\text { of IEI. } \\
\% \text { Response rate } \\
71.5 \% \text { (732 people) } \\
\text { Analyzed } \\
\text { G1: } 571 \text { people } \\
\text { men: } 194 \text {; female: } 377 \\
\text { mean age } 48.1 \text { (12.4) } \\
\text { G2: } 161 \text { people } \\
\text { men: } 21 \text {; female: } 140 \\
\text { mean age } 53 \text { (SD } \\
\text { 10.6) } \\
\text { G2> G1 age (p } \\
\text { p } \leq 0,001 \text { ) }\end{array}$ & $\begin{array}{l}\text { Interventions } \\
\text { Auto shipping questionnaires } \\
\text { by mail. } \\
\text { Evaluation depression by } \\
\text { Symptom Checklist } 92 \text { (SCL- } \\
\text { de subscale) } \\
\text { Social support perceived } \\
\text { (ASP) } \\
\text { Recent life events (AVR) } \\
\text { Scales: } \\
\text { Symptoms of Central Nervous } \\
\text { System (ESSNC). Score } \\
\text { between } 0 \text { and } 8 . \\
\text { Mucous symptoms (ESM). } \\
\text { Score between } 0 \text { and } 6 . \\
\text { chemical hypersensitivity } \\
\text { (EHQ). Score between } 0 \text { and } \\
33 . \\
\text { Implications for Social } \\
\text { Activities (ECAS). } \\
\text { Score between } 0 \text { and } 14 . \\
\text { Statistic analysis } \\
\text { Comparative analysis } \\
\text { Correlation analysis }\end{array}$ & $\begin{array}{l}\text { Comparative } \\
\text { SCL-de:> symptoms in } \mathrm{G} 2(\mathrm{p} \leq 0,001) \\
\text { ASP: <social support perceived in } \mathrm{G} 2 \\
(\mathrm{p} \leq 0,001) \\
\text { AVR: No differences between groups } \\
\text { ESSNC: }>\text { involvement in } \mathrm{G} 2(\mathrm{p} \leq 0,001) \\
\text { ESM: }>\text { involvement in } \mathrm{G} 2(\mathrm{p} \leq 0,001) \\
\text { EHQ:> involvement in } \mathrm{G} 2(\mathrm{p} \leq 0,001) \\
\text { ECAS:> involvement in } \mathrm{G} 2(\mathrm{p} \leq 0,001) \\
\text { Correlation between variables } \\
\text { Significant positive correlation between } \\
\text { moderate: } \\
\text { ESM and ESP SNC } \\
\text { ESM and ECAS } \\
\text { ESM and EHQ } \\
\text { ESSNC and ECAS } \\
\text { ESSNC and EHQ } \\
\text { ECAS and EHQ } \\
\text { Remaining positive correlations are low or } \\
\text { very low, all significant except between: } \\
\text { ECAS and AVR } \\
\text { ESSNC and Age } \\
\text { ASP and Age } \\
\text { Very low negative correlation between: } \\
\text { SCL-de and Age } \\
\text { AVR and age ( } \mathrm{p}=0.01)\end{array}$ & $\begin{array}{l}\text { The association between } \\
\text { IIA and psychological } \\
\text { disorders (depression } \\
\text { symptoms) is not } \\
\text { explained by known risk } \\
\text { factors for severe } \\
\text { depression, such as social } \\
\text { support and recent life } \\
\text { events } \\
\text { The differences between } \\
\text { people and group patients } \\
\text { in the study suggest that } \\
\text { psychological } \\
\text { disturbances may be a } \\
\text { risk factor or part of the } \\
\text { most serious states of } \\
\text { IEI, possibly adding to } \\
\text { the level of disability, ie, } \\
\text { the social and labor. }\end{array}$ \\
\hline
\end{tabular}

\begin{tabular}{|c|c|c|c|c|c|}
\hline REFERENCE & STUDY & POPULATION & $\begin{array}{l}\text { INTERVENTION / } \\
\text { COMPARISON }\end{array}$ & $\begin{array}{l}\text { CLINIC / } \\
\text { COMORBIDITY }\end{array}$ & CONCLUSIONS \\
\hline $\begin{array}{l}\text { Nordin } \text { et al. } \\
2013^{37} \text {, United } \\
\text { Kingdom }\end{array}$ & $\begin{array}{l}\text { Objetive } \\
\text { Investigate perceived } \\
\text { stress, focusing on } \\
\text { emotional factors and } \\
\text { environmental } \\
\text { sensitivity to odors in } \\
\text { people with very high } \\
\text { sensitivity to noise } \\
\text { compared to people } \\
\text { with low sensitivity to } \\
\text { noise. } \\
\text { Study design } \\
\text { Transversal study. } \\
\text { Period of realization } \\
\text { They do not tell. }\end{array}$ & $\begin{array}{l}\text { Recruitment } \\
134 \text { male college students. } \\
\text { Weinstein's answer questionnaire Noise } \\
\text { Sensitivity Scale (NSS) (classifies noise } \\
\text { sensitivity as negative emotional } \\
\text { reactions and behavioral disturbances } \\
\text { caused by noise) } \\
\text { Range between } 1 \text { and } 105 \text { (a> score> } \\
\text { sensitivity) } \\
\text { Groups } \\
\text { Group } 1 \text { (G1): } \\
16 \text { people } \\
\text { high sensitivity } \\
\text { Average age: } 23 \text { a (DE 2.5) } \\
\text { NSS Average Score: } 85.4 \text { (SD 7.5) } \\
\text { Group } 2 \text { (G2): } \\
16 \text { people } \\
\text { low sensitivity } \\
\text { Average age: the 24th (SD 2.9) } \\
\text { NSS Average Score: } 55.2 \text { (SD 6.1) }\end{array}$ & $\begin{array}{l}\text { Interventions } \\
\text { Perceived stress } \\
\text { Perceived Stress Questionnaire } \\
\text { (PSQ): Range between } 0 \text { and } 1 \\
\text { (> value> stress) } \\
\text { Sensitivity to odors } \\
\text { Chemical Sensitivity Scale } \\
\text { (CSS): range between } 1 \text { and } 105 \\
\text { (> value> sensitivity) } \\
\text { Statistic analysis } \\
\text { Analysis of variance } \\
\text { Spearman correlation }\end{array}$ & $\begin{array}{l}\text { Perceived stress }(\text { PSQ): } \\
\text { G1> G2 stress }(p>0.05) \\
\text { Sensitivity to odors }(\text { CSS): } \\
\text { G1> G2 sensitivity }(p>0.05) \\
\text { Correlation between: } \\
\text { NSS and PSQ: } 0.35 \text { low } \\
\text { positive correlation ( } p<0.05) \\
\text { NSS and CSS: } 0,48 \\
\text { moderate positive correlation } \\
\text { ( }<<0.01 \text { ) } \\
\text { PSQ and CSS: } 0,58 \\
\text { moderate positive correlation } \\
(p<0.001)\end{array}$ & $\begin{array}{l}\text { The question of } \\
\text { whether the } \\
\text { relationship between } \\
\text { susceptibility to } \\
\text { noise and odors } \\
\text { reflects a general } \\
\text { environmental } \\
\text { sensitivity arises }\end{array}$ \\
\hline
\end{tabular}




\section{Diagnosis}

\begin{tabular}{|c|c|c|c|c|}
\hline REFERENCE & STUDY / POPULATION & INDICATOR & RESULTS & CONCLUSIONS / COMMENTS \\
\hline $\begin{array}{l}\text { Mena et al. } \\
2013^{41}, \text { Spain }\end{array}$ & $\begin{array}{l}\text { Objetive } \\
\text { QEESI translate and adapt to Castilian and } \\
\text { Spanish population and analyze their reliability in } \\
\text { people with MCS diagnosed in Tertiary Hospital. } \\
\text { Study design } \\
\text { Transversal study. } \\
\text { Sample } \\
\text { Group } 1 \text { (G1): } 77 \text { ( } 74 \text { women) people diagnosed } \\
\text { with MCS. } \\
\text { Average age: } 54.2 \text { years (SD 6.5). } \\
\text { Group } 2 \text { (G2): } 154 \text { people. Recruited outpatient } \\
\text { without criteria MCS. } \\
\text { Average age: } 52.3 \text { years (SD } 8.7 \text { ). }\end{array}$ & $\begin{array}{l}\text { Interventions } \\
\text { Translation QEESI the } \\
\text { Castilian, back } \\
\text { translation, committee } \\
\text { discussion and piloting. } \\
\text { Statistic analysis } \\
\text { For valuation of internal } \\
\text { consistency of the scales: } \\
\text { Cronbach } \alpha \text {. }\end{array}$ & $\begin{array}{l}\text { Cronbach } \boldsymbol{\alpha} \text { of: } \\
\text { Chemical intolerance: } 0,81 \\
\text { Other intolerances. } 0.85 \\
\text { Severity of symptoms: } 0,81 \\
\text { Impact daily life activities: } 0.87\end{array}$ & $\begin{array}{l}\text { This adapted version of QEESI } \\
\text { presents, in terms of reliability, good } \\
\text { internal consistency. } \\
\text { COMMENTS } \\
\text { Prospective longitudinal studies } \\
\text { considered necessary to calculate the } \\
\text { ROC curves (AUC) in order to } \\
\text { establish cutoff points for each scale } \\
\text { Propose other studies to design } \\
\text { instruments with better ability to } \\
\text { diagnose MCS. }\end{array}$ \\
\hline
\end{tabular}

\begin{tabular}{|c|c|c|c|c|}
\hline REFERENCE & STUDY / POPULATION & INDICATOR & RESULTS & CONCLUSIONS / COMMENTS \\
\hline $\begin{array}{l}\text { Skovbjerg et al. } \\
2012 b^{38} \\
\text { Denmark }\end{array}$ & $\begin{array}{l}\text { Objetive } \\
\text { Evaluate the translation of QEESI for } \\
\text { Danish population. } \\
\text { Study design } \\
\text { Transversal study. } \\
\text { Period of realization } \\
\text { See below. } \\
\text { Involved } \\
\text { Group } 1 \text { (G1): } \\
\text { 1st questionnaire Shipping: } 2,000 \\
\text { people (between } 18 \text { and } 69 \text { years) } \\
\text { 2nd questionnaire Shipping: } 200 \\
\text { Danish Civil Registration come } \\
\text { (January } 2010 \text { ) } \\
\text { Group } 2 \text { (G2): } \\
\text { 1st questionnaire Shipping: } 315 \text { people. } \\
\text { 183 after contact with Danish Research } \\
\text { Centre for Chemical Sensitivities } \\
\text { (between January } 2006 \text { and January } \\
\text { 2010). 132 people diagnosed with } \\
\text { MCS hospital between January } 1990 \\
\text { and January 2009 } \\
\text { 2nd questionnaire Shipping: } 140 \\
\text { people. }\end{array}$ & $\begin{array}{l}\text { Interventions } \\
\text { Translation with piloting. } \\
\text { Questionnaire sent } 2 \text { times } \\
\text { (starts and two months) } \\
\text { 1st questionnaire } \\
\text { responses: } \\
\text { Global: } 64.5 \% \\
\text { G1: } 65.3 \% \text { (1305/2000) } \\
\text { G2: } 60 \% \text { (189/315) } \\
\text { 2nd questionnaire } \\
\text { responses: } \\
\text { G1: } 61 \% \text { (122/200) } \\
\text { G2: } 80 \% \text { (112/140) } \\
\text { Statistic analysis } \\
\text { Reliability: } \\
\text { Internal consistency: } \\
\text { Cronbach } \alpha \\
\text { Test-retest (same test } \\
\text { people at two different } \\
\text { times - Home and } 2 \\
\text { months): Pearson } \\
\text { Correlation } \\
\text { Sensitivity and } \\
\text { specificity: } \\
\text { Area under the curve }\end{array}$ & $\begin{array}{l}\text { Internal consistency: } \\
\text { G1: between } 0.64 \text { and } 0.94 \text { (x age } \\
\text { groups) } \\
\text { G2: between } 0,83 \text { and } 0,91 \\
\text { Test-retest: } \\
\text { Between } 0.84 \text { and } 0.96 \text {. Positive } \\
\text { correlation between high and very } \\
\text { high (p <0.05) } \\
\text { Overall sensitivity (S): } 92.1 \% \\
\text { overall specificity (E): } 93.1 \% \\
\text { Intolerance Scale Chemistry (IQ): } \\
\text { S: } 89.3 \% \text { (for cutting point scale 47) } \\
\text { E: } 89.4 \% \text { (for cutting point scale 47) } \\
\text { Scale impact on daily life (IVD): } \\
\text { S: } 91.0 \% \text { (for cutting point scale 21) } \\
\text { E: } 90.9 \% \text { (for cutting point scale 21) } \\
\text { Combined use IQ and IVD: } \\
\text { S: } 92.1 \% \text { (35 point IQ cut and IVD } \\
\text { 14) } \\
\text { E: } 91.8 \% \text { (35 point IQ cut and IVD } \\
\text { 14) }\end{array}$ & $\begin{array}{l}\text { Danish translation of QEESI shows good } \\
\text { reliability and validity. } \\
\text { Recommend using scales "Chemical } \\
\text { Intolerance" and "impact on activities of } \\
\text { daily living" as it is a shorter alternative } \\
\text { with good S and E. }\end{array}$ \\
\hline
\end{tabular}

Physical, psychological and social impact. Quality of life

\begin{tabular}{|c|c|c|c|c|c|}
\hline REFERENCE & STUDY & POPULATION & INTERVENTION & IMPLICATION & $\begin{array}{l}\text { CONCLUSIONS / } \\
\text { COMMENTS }\end{array}$ \\
\hline $\begin{array}{l}\text { Gibson } \text { et al. } \\
2011^{42} \text {, U.S. }\end{array}$ & $\begin{array}{l}\text { Objetive } \\
\text { Investigate the } \\
\text { long-term impact } \\
\text { of the MCS in } \\
\text { the lives of } \\
\text { patients with } \\
\text { MCS, focusing } \\
\text { on relationships. } \\
\text { Study design } \\
\text { Qualitative } \\
\text { study. }\end{array}$ & $\begin{array}{l}\text { Inclusion criteria } \\
\text { MCS minimum of } 5 . \\
\text { Recruitment } \\
\text { Advertisements. } \\
\text { Sample } \\
15 \text { women } \\
11 \text { men } \\
\text { Characteristics } \\
\text { Average age: } 59 \text { years } \\
\text { Range: } 31-82 \\
\text { Symptoms Average } \\
\text { duration: } 23.5 \text { years } \\
\text { Range: } 5 \text { to } 51 \text { years }\end{array}$ & $\begin{array}{l}\text { Methodology } \\
\text { Telephone interview: } \\
\text { Yes, and how their relationships with } \\
\text { others are affected by having MCS } \\
\text { The way they thought they were } \\
\text { perceived by others and if there are } \\
\text { misconceptions about disability } \\
\text { If you have ever felt disconnected } \\
\text { from society because MCS } \\
\text { Reading, transcription, discussion } \\
\text { group and re-reading. } \\
\text { Grouping content by topics: } \\
\text { Adequacy of spaces ("primacy of } \\
\text { spatiality") }\end{array}$ & $\begin{array}{l}\text { Adequacy of spaces: } \\
\text { Social and occupational isolation } \\
\text { Need to take many precautions to relate. } \\
\text { a cultural effort is needed to create safe } \\
\text { spaces (work, cinema, transport, etc.) } \\
\text { where you can interact with others. } \\
\text { Reaching out to others: } \\
\text { Incomprehension, disbelief, ignorance, not } \\
\text { ill. } \\
\text { Loneliness, guilt by high demand for } \\
\text { maintaining relationships. } \\
\text { One solution may be to educate the general } \\
\text { population about MCS, although not all } \\
\text { share. }\end{array}$ & $\begin{array}{l}\text { Described under } \\
\text { discussion: } \\
\text { Respondents living very } \\
\text { different lives and see } \\
\text { the world from a } \\
\text { markedly different than } \\
\text { those without MCS } \\
\text { perspective. } \\
\text { Require constant } \\
\text { precautions, tendency to } \\
\text { social isolation, it } \\
\text { emphasizes the } \\
\text { importance of the } \\
\text { adequacy of the spaces. }\end{array}$ \\
\hline
\end{tabular}


Review of the Available Scientific Evidence on Multiple Chemical Sensitivity Syndrome

\begin{tabular}{|c|c|c|c|c|c|}
\hline REFERENCE & STUDY & POPULATION & INTERVENTION & IMPLICATION & $\begin{array}{l}\text { CONCLUSIONS / } \\
\text { COMMENTS }\end{array}$ \\
\hline & & & $\begin{array}{l}\text { Reaching out to } \text { others ("reaching } \\
\text { for others") } \\
\text { Living in a different world (includes } \\
\text { previous issues) }\end{array}$ & $\begin{array}{l}\text { Internet and telephone possible to maintain } \\
\text { contact with people. } \\
\text { Living in a different world: } \\
\text { They feel the world outside as mere } \\
\text { observers. }\end{array}$ & $\begin{array}{l}\text { Health care providers } \\
\text { must broaden their } \\
\text { perspectives and create } \\
\text { safe spaces for people } \\
\text { with MCS. }\end{array}$ \\
\hline
\end{tabular}

\begin{tabular}{|c|c|c|c|c|c|}
\hline REFERENCE & STUDY & POPULATION & INTERVENTION & REPERCUSIONES & $\begin{array}{l}\text { CONCLUSIONS / } \\
\text { COMMENTS }\end{array}$ \\
\hline $\begin{array}{l}\text { Soderholm } \text { et } \\
\text { al. } 2011^{43} \text {, } \\
\text { Sweden }\end{array}$ & $\begin{array}{l}\text { Objetive } \\
\text { Clarify how } \\
\text { individuals } \\
\text { experience } \\
\text { living with } \\
\text { sensory } \\
\text { hyperactivity } \\
\text { disorder (HRS), } \\
\text { the impact on } \\
\text { accessibility, } \\
\text { economic } \\
\text { security and } \\
\text { social relations. } \\
\text { Study design } \\
\text { Qualitative } \\
\text { study. }\end{array}$ & $\begin{array}{l}\text { According to the } \\
\text { authors, time is a } \\
\text { form of intolerance } \\
\text { odors, including } \\
\text { MCS and IEI. } \\
\text { Inclusion criteria } \\
\text { HOURS be } \\
\text { diagnosed by a } \\
\text { physician with the } \\
\text { provocative test of } \\
\text { capsaicin, be } \geq 18 \\
\text { years old and live } \\
\text { in Sweden. } \\
\text { Recruitment } \\
\text { Ad on specific } \\
\text { web. } \\
\text { Sample } \\
16 \text { volunteers } \\
12 \text { women met } \\
\text { inclusion criteria } \\
\text { Characteristics } \\
\text { Age range: } 23 \text { to } \\
64 a \\
\text { Symptoms range } \\
\text { duration: } 1 \text { to } 20 a\end{array}$ & $\begin{array}{l}\text { Methodology } \\
\text { I relate answering three } \\
\text { written questions: How } \\
\text { do you experience the } \\
\text { impact of this condition } \\
\text { in its accessibility to } \\
\text { society; in their social } \\
\text { relationships; in } \\
\text { economic security? } \\
\text { They use a scale } \\
\text { chemical-sensory } \\
\text { hyperreactivity } \\
\text { sensitivity to quantify } \\
\text { emotional reactions and } \\
\text { behavioral disorders in } \\
\text { everyday life by odors. } \\
\text { Cutoff for HRS is } \geq \mathbf{4 3} \\
\text { points. } \\
\text { Groupings topics: } \\
\text { Accessibility } \\
\text { Economic security } \\
\text { Social relationships }\end{array}$ & $\begin{array}{l}\text { All they obtained on the sensitivity scale chemical-HRS } \\
\geq 43 \\
\text { Media } \mathbf{5 2 . 3} \text { (range 44-55) } \\
\text { Subjects identified categories: } \\
\text { Accessibility: } \\
\text { Take transport is difficult. } \\
\text { Visit public buildings and facilities is difficult. } \\
\text { Finding a suitable place to live is almost impossible. } \\
\text { Economic security: } \\
\text { Reduction in revenue due to the difficulty of living. } \\
\text { Increased expenses have HRS } \\
\text { Lack of support from the authorities. } \\
\text { Difficulty managing finances. } \\
\text { Social relationships: } \\
\text { Socializing has become difficult and traumatic. } \\
\text { Limitation for social activities. } \\
\text { Getting support from some people knowing that this limits } \\
\text { people. } \\
\text { Refusing to change their own social interaction. } \\
\text { Six common themes for the three content areas were } \\
\text { identified: } \\
\text { Limitation to participate in society } \\
\text { Obligation to behave inconsistently with personality } \\
\text { Experience lack of understanding and respect for others } \\
\text { experiencing insecurity } \\
\text { Being dependent on others } \\
\text { Forced to choose between plague and cholera. Alternative } \\
\text { sometimes are equally negative. }\end{array}$ & $\begin{array}{l}\text { If you choose to avoid } \\
\text { odors: } \\
\text { Determinants of health } \\
\text { (accessibility, financial } \\
\text { security, social relations) } \\
\text { are adversely affected } \\
\text { If they decide exposed to } \\
\text { odors: } \\
\text { Determinants of health can } \\
\text { be affected positively, but } \\
\text { will get sick with } \\
\text { symptoms HRS, with the } \\
\text { loss of livestock, not } \\
\text { physically able to work, } \\
\text { travel and participate in } \\
\text { activities and social } \\
\text { gatherings. }\end{array}$ \\
\hline
\end{tabular}

\begin{tabular}{|c|c|c|c|}
\hline REFERENCE & STUDY & RESULTS & $\begin{array}{l}\text { CONCLUSIONS / } \\
\text { COMMENTS }\end{array}$ \\
\hline $\begin{array}{l}\text { Genuis, 2013 }{ }^{45} \text {, } \\
\text { Canada }\end{array}$ & $\begin{array}{l}\text { Objetive } \\
\text { Provide information on the management of MCS. } \\
\text { Study design } \\
\text { Review } \\
\text { Search period } \\
\text { Not specified } \\
\text { Revised databases } \\
\text { MEDLINE, books, magazines toxicology, conference } \\
\text { papers, government publications and magazines } \\
\text { environmental health. }\end{array}$ & $\begin{array}{l}\text { Desensitization immunotherapy } \\
\text { Steroids. Alternative that is not first line. } \\
\text { Cognitive therapy and neuronal Retraining: } \\
\text { technique from the premise of neuroplasticity and } \\
\text { involvement of the limbic system in the MCS, } \\
\text { proposes a training by instructions to restructure } \\
\text { and modify the operation of the limbic system. } \\
\text { RESULTS variables without a clear effectiveness. } \\
\text { Moving away from social activities (avoid } \\
\text { chemicals and create a free-living chemicals) }\end{array}$ & $\begin{array}{l}\text { Physiological treatments appear } \\
\text { to have superior and sustainable } \\
\text { RESULTS compared to } \\
\text { psychological therapies. } \\
\text { As a preventable and reversible } \\
\text { condition, people with MCS } \\
\text { need of attention directed to } \\
\text { avoid exposure to triggers and } \\
\text { attention directed to inform } \\
\text { these people substances. }\end{array}$ \\
\hline
\end{tabular}

\section{Therapeutic management and prevention}

\begin{tabular}{|c|c|c|c|c|c|}
\hline REFERENCE & STUDY & POPULATION & INTERVENTION & RESULTS & $\begin{array}{l}\text { CONCLUSIONS / } \\
\text { COMMENTS }\end{array}$ \\
\hline $\begin{array}{l}\text { Ralph } \text { et al. } \\
2011^{46} \text {, } \\
\text { Luxembourg } \\
\text { and France } \\
\text { (Study } \\
\text { addressing }\end{array}$ & $\begin{array}{l}\text { Objetive } \\
\text { Tracking a } \\
\text { case since his } \\
\text { diagnosis, } \\
\text { investigating } \\
\text { changes in }\end{array}$ & $\begin{array}{l}\text { Woman } \\
\text { Social worker } \\
\text { Occupational exposure } \\
\text { unlikely } \\
\text { From } 2004 \text { to August } \\
\text { 2007: }\end{array}$ & $\begin{array}{l}\text { Diagnosing triggers products and reduce or eliminate symptoms of } \\
\text { exposure. } \\
\text { Identified home products: } \\
\text { Biocidal products (17 products): Azaconazole, chlorothalonil, } \\
\text { chlorpyrifos, 4,4'-DDE, 4,4'-DDT, dichlofluanid, dieldrin, } \\
\text { endosulfan, Eulan, adjoin, methoxychlor, pentachlorophenol, }\end{array}$ & $\begin{array}{l}\text { Onset of } \\
\text { symptoms after } \\
\text { exposure to } \\
\text { permethrin. } \\
\text { syndromes } \\
\text { solvents and }\end{array}$ & $\begin{array}{l}\text { The appearance of } \\
\text { symptoms of MCS } \\
\text { after accidental } \\
\text { exposure to permethrin } \\
\text { corroborates the cause- } \\
\text { effect relationship. }\end{array}$ \\
\hline
\end{tabular}


Alberto Frutos Pérez-Surio et al.

\begin{tabular}{|c|c|c|c|c|c|}
\hline $\begin{array}{l}\text { prevention and } \\
\text { therapeutic } \\
\text { measure) }\end{array}$ & $\begin{array}{l}\text { clinical } \\
\text { regarding } \\
\text { changes in } \\
\text { exposure. } \\
\text { Design } \\
\text { Descriptive, } \\
\text { apropos of a } \\
\text { case. } \\
\text { Period of } \\
\text { realization } \\
\text { From } 2007 .\end{array}$ & $\begin{array}{l}\text { baffling symptoms } \\
\text { with multiple medical } \\
\text { research } \\
\text { Severe pain and } \\
\text { headaches with visual } \\
\text { impairment } 24-28 \\
\text { hours after furniture } \\
\text { restoration weekend. } \\
\text { improvements and } \\
\text { exacerbations alternate } \\
\text { according restatements }\end{array}$ & $\begin{array}{l}\text { propiconazole, tetrachlorvinphos, tolyfluanid and tribromophenol, } \\
\text { tebuconazole } \\
\text { Pyrethrins (8 products): Permethrin, cyfluthrin, cypermethrin, } \\
\text { deltamethrin, fenvalerate, phenothrin, tetramethrin and piperonyl } \\
\text { butoxid } \\
\text { Flame retardants (8 products): 2-ethylhexyl-diphenyl-phosphate } \\
\text { EHDPP, tributyl-phosphate TBP, TPP triphenyl-phosphate, tris (2- } \\
\text { butoxyethyl) -phosphate TBEP, tris (2-chloroethyl) -phosphate } \\
\text { TCEP, tris (3-chloropropyl) -phosphate TCPP; tris (1,3-dichloro-2- } \\
\text { propyl) -phosphate TDCP or TDCPP and tris (2-ethylhexyl) - } \\
\text { phosphate TEHP. } \\
\text { Bed mattress: very high levels of permethrin (30 mg / kg of foam). } \\
\text { Notable changes to the clinic as exposure to permethrin mattress. }\end{array}$ & $\begin{array}{l}\text { olfactory } \\
\text { syndrome are } \\
\text { excluded } \\
\text { (Permethrin is } \\
\text { odorless) } \\
\text { The replacement } \\
\text { mattress marked } \\
\text { improvement in } \\
\text { symptomato- } \\
\text { logy one woman }\end{array}$ & $\begin{array}{l}\text { Environmental } \\
\text { toxicological } \\
\text { investigations ensured } \\
\text { a diagnosis of existing } \\
\text { at a time that could } \\
\text { implement treatment } \\
\text { measures designed to } \\
\text { prevent re-exposure } \\
\text { substances. }\end{array}$ \\
\hline
\end{tabular}

\begin{tabular}{|c|c|c|c|c|c|}
\hline REFERENCE & STUDY & POPULATION & INTERVENTION & RESULTS & $\begin{array}{l}\text { CONCLUSIONS / } \\
\text { COMMENTS }\end{array}$ \\
\hline $\begin{array}{l}\text { Mischley et al. } \\
2013^{48} \text {, U.S. }\end{array}$ & \begin{tabular}{|l} 
Objetive \\
Describe \\
RESULTS \\
provided by \\
people with \\
prescription \\
intranasal \\
reduced \\
glutathione \\
(inGSH).
\end{tabular} & $\begin{array}{l}\text { Inclusion criteria } \\
\text { You have received } \\
\text { one or more } \\
\text { prescriptions Ings } \\
\text { Between April } \\
2009 \text { and April } \\
2011 . \\
\text { Recruitment } \\
\text { Pharmaceutical } \\
\text { base data. } \\
\text { Sample } \\
\mathbf{3 0 0} \text { individuals } \\
\text { selected for } \\
\text { randomization }\end{array}$ & $\begin{array}{l}\text { Mailed questionnaire. } \\
\text { Individual } \\
\text { perceptions about: } \\
\text { Adverse effects } \\
\text { Health benefits }\end{array}$ & $\begin{array}{l}\text { Response rate: } 23.3 \%(\mathrm{n}=70) \\
\text { Confirm prescription } 94.3 \%(\mathrm{n}=66) \\
\text { MCS } \\
\text { Prescription inGSH MCS: } 42 \%(\mathrm{n}=29) \\
\text { InGSH usage time in MCS - months: } \\
\text { Median (p25; } 75) ; 32.5(16 ; 65) \\
\text { negative effects described: } 20.7 \%(\mathrm{n}=6) \\
\text { Health benefits described: } 62.1 \%(\mathrm{n}=18) \\
\text { Improving energy: } 17.2 \%(\mathrm{n}=5) \\
\text { They feel good: } 31.0 \%(\mathrm{n}=9) \\
\text { Improved sense of smell: } 10.3 \%(\mathrm{n}=3) \\
\text { Amelioration of symptoms of MCS: } 44.8 \%(\mathrm{n}=13) \\
\downarrow \text { head pain often: } 13.8 \%(\mathrm{n}=4) \\
\downarrow \text { sinusitis: } 13.8 \%(\mathrm{n}=4) \\
\downarrow \text { otitis: } 3.4 \%(\mathrm{n}=1) \\
\text { Adverse effects } \\
\text { Irritation of sinuses or nasal passage: } 31 \%(\mathrm{n}=9) \\
\text { Headaches: } 20.7 \%(\mathrm{n}=6) \\
\text { Worsening symptoms MCS: } 3.4 \%(\mathrm{n}=1) \\
\text { Epistaxis: } 13.8 \%(\mathrm{n}=4)\end{array}$ & $\begin{array}{l}\text { inGSH is easy to administer, } \\
\text { with few adverse effects and } \\
\text { perceived health } \\
\text { improvements } \\
\text { Future studies could be } \\
\text { aimed at determining whether } \\
\text { the individual perception of } \\
\text { improvement can be } \\
\text { objectively verified and if } \\
\text { these benefits can be inferred } \\
\text { to larger population. }\end{array}$ \\
\hline
\end{tabular}

\begin{tabular}{|l|l|l|l|l|l|}
\hline REFERENCE & STUDY & POPULATION & INTERVENTION & RESULTS & CONCLUSIONS / COMMENTS \\
\hline $\begin{array}{l}\text { Waddick, 2011 } \\
\text { U.S. }\end{array}$ & $\begin{array}{ll}\text { Objetive } \\
\text { Analyze residential areas } \\
\text { to reduce exposure to } \\
\text { contaminants in MCS } \\
\text { vulnerable population. } \\
\text { Study design } \\
\text { Case Studies } \\
\text { Period of realization } \\
\text { Not specified }\end{array}$ & $\begin{array}{l}\text { Two healthy } \\
\text { residential areas }\end{array}$ & $\begin{array}{l}\text { interviews: } \\
\text { 1-Personal } \\
\text { 2-Phone } \\
\text { Visits to residential } \\
\text { areas }\end{array}$ & $\begin{array}{l}\text { Difficulties in finding safe housing } \\
\text { Planning actions (to address these challenges) } \\
\text { Role of people } \\
\text { Economy and finance staff } \\
\text { Safe maintenance and property management } \\
\text { (housing) } \\
\text { Access to affordable and safe housing for } \\
\text { vulnerable populations. }\end{array}$ & $\begin{array}{l}\text { In the absence of etiological } \\
\text { treatments, more research is needed } \\
\text { on ways to create and sustain } \\
\text { healthy residential areas, understand } \\
\text { and reduce sources of exposure that } \\
\text { initiate and trigger the MCS and } \\
\text { learn from experiences and } \\
\text { strategies used in other countries. }\end{array}$ \\
\hline
\end{tabular}

Table 2. Excluded studies

\begin{tabular}{|l|l|}
\hline REFERENCE & EXCLUSION CRITERIA \\
\hline EPIDEMIOLOGY & It does not fit the study population. \\
\hline Baliatsas et al. 2014, Holland & It does not fit the study population. \\
\hline CLINICAL MANIFESTATIONS AND COMORBIDITY \\
\hline Baliatsas et al. 2014, Holland & Narrative review. \\
\hline Genuis 2013, Canada &
\end{tabular}


Review of the Available Scientific Evidence on Multiple Chemical Sensitivity Syndrome

\begin{tabular}{|c|c|}
\hline REFERENCE & EXCLUSION CRITERIA \\
\hline Tran et al. 2013b, Denmark & Study protocol. \\
\hline De Luca et al. 2011, Italy and Malaysia & Narrative review. \\
\hline Goldstein et al. 2011, U.S & It does not address the intervention of interest. \\
\hline Leer et al. 2011, Holland & It does not address the intervention of interest. \\
\hline Skovbjerg et al. 2012c, Denmark & It does not address the intervention of interest. \\
\hline Skovbjerg et al. 2012d, Denmark & It does not fit the study population. \\
\hline Nogué et al. 2011, Spain & Narrative review. \\
\hline \multicolumn{2}{|l|}{ MCS DIAGNOSIS } \\
\hline Dupas et al. 2013, France & Narrative review. \\
\hline Mazzatenta et al. 2013, Italy & It does not address the intervention of interest. \\
\hline Nordin et al. 2013, United Kingdom & It does not address the intervention of interest. \\
\hline Tran et al. 2013a, Denmark & It does not address the intervention of interest. \\
\hline Baliatsas et al. 2012, Holland & It does not fit the study population. \\
\hline Fujimori et al. 2012, Japan & It does not address the intervention of interest. \\
\hline \multicolumn{2}{|c|}{ PHYSICAL, PSYCHOLOGICAL AND SOCIAL IMPACT. QUALITY OF LIFE } \\
\hline Baliatsas et al. 2014, Holland & It does not fit the study population. \\
\hline Heinonen-Guzejev et al. 2012, Finland & It does not address the intervention of interest. \\
\hline Skovbjerg et al. 2012b, Denmark & It does not address the intervention of interest. \\
\hline Skovbjerg et al. 2012c, Denmark & It does not fit the study population. \\
\hline Skovbjerg et al. 2012d, Denmark & It does not address the intervention of interest. \\
\hline De Luca et al. 2011, Italy and Malaysia & Narrative review. \\
\hline Waddick 2011, U.S & It does not address the intervention of interest. \\
\hline \multicolumn{2}{|l|}{ PREVENTION AND TREATMENT } \\
\hline Baliatsas et al. 2014, Holland & It does not fit the study population. \\
\hline Tran et al. 2013b, Denmark & Study protocol. \\
\hline Araki et al. 2012, Japan & It does not address the intervention of interest. \\
\hline Hauge et al. 2012, Denmark & Study protocol. \\
\hline De Luca et al. 2011, Italy and Malaysia & Narrative review. \\
\hline Stoppe et al. 2011, Germany & It does not fit the study population. \\
\hline Williams et al. 2011, Canada & It does not address the intervention of interest. \\
\hline Witthoft et al. 2013, UK and Germany & It does not address the intervention of interest. \\
\hline Zaitseva et al. 2011, Russia & It does not fit the study population. \\
\hline
\end{tabular}




\section{IMAGES:}

PRISMA 2009 Flow Diagram
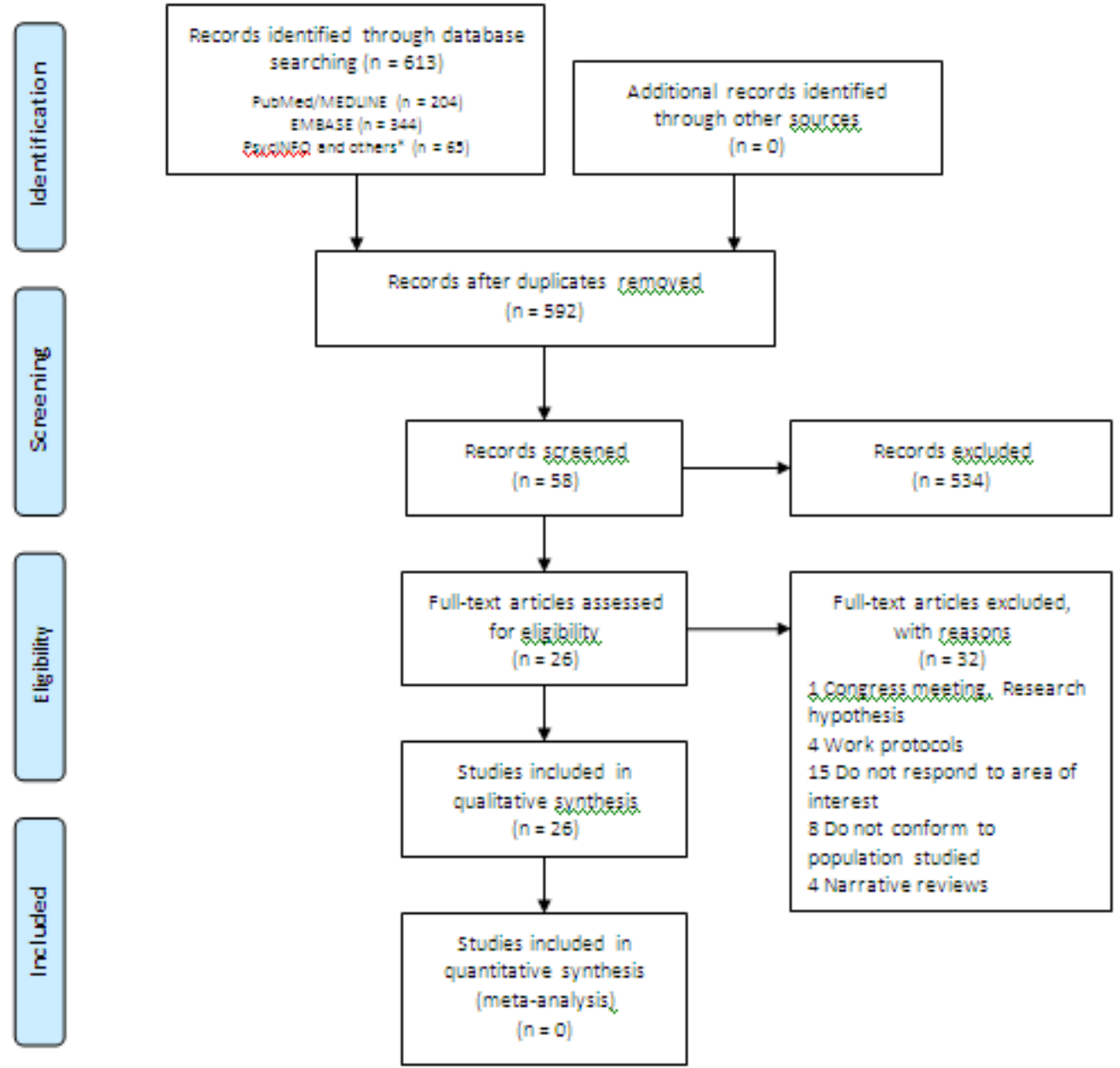

REFERENCES

${ }^{1}$ Bartha L, Baumzweiger W, Buscher DS, Callender T, Dahal KA, Davidoff A et al. Multiple chemical sensitivity: a 1999 consensus. Arch Environ Health 1999; 54: 147-9

${ }^{2}$ Miller CS. Multiple chemical sensitivity syndrome. Occup Environ Med. 1995; 37:1323.

${ }^{3}$ Regal Ramos RJ. Can we rule out that fibromyalgia, chronic fatigue syndrome and multiple chemical sensitivity are psychosomatic diseases? Semergen. 2015 Oct;41(7):349-53.

${ }^{4}$ Godás Sieso T, Nogué Xarau S, Salamero Baró M, Fernandez Solá J. Psychopathologic status in patients with chronic fatigue syndrome, associated or not with multiple chemical sensitivity. Med Clin (Barc). 2014 Nov 18;143(10):467-8.

${ }^{5}$ Staudenmayer H. Idiopathic environmental intolerances (IEI): Myth and reality. Toxicol Lett. 2001 120(1-3): 333-42.

${ }^{6}$ Bolt H.M. Kiesswetter E. Is multiple chemical sensitivity a clinically defined entity. Toxicol Lett. 2002 128(1-3): 99-106.

${ }^{7}$ Winder C. Mechanisms of multiple chemical sensitivity. Toxicol Lett. 2002 128(1-3): 85-97.

${ }^{8}$ Corsini E. Kimber I. Factors governing susceptibility to chemical allergy. Toxicol Lett. 2007 168(3): $255-9$.

${ }^{9}$ Baliatsas C, van Kamp I, Hooiveld M, Yzermans J, Lebret E. Comparing non- specific physical symptoms in environmentally sensitive patients: prevalence, duration, functional status and illness behavior. J Psychosom Res. 2014 May;76(5):405-13.

10 Azuma K, Uchiyama I, Katoh T, Ogata H, Arashidani K, Kunugita N. Prevalence and Characteristics of Chemical Intolerance: A Japanese Population-Based Study. Arch Environ 
Occup Health. 2015 Nov 2;70(6):341-53.

${ }^{11}$ Dantoft TM, Andersson L, Nordin S, Skovbjerg S: Chemical intolerance. Curr Rheumatol Rev 11(2):167-184, 2015

${ }^{12}$ Frías Á: Idiopathic environmental intolerance: A comprehensive and up-to-date review of the literature. CNS 1(1):31-37, 2015

${ }^{13}$ De Luca C, Gugliandolo A, Calabrò C, Currò M, Ientile R, Raskovic D, Korkina L, Caccamo D. Role of polymorphisms of inducible nitric oxide synthase and endothelial nitric oxide synthase in idiopathic environmental intolerances. Mediators Inflamm. 2015;2015:245308.

${ }^{14}$ Hauge CR, Rasmussen A, Piet J, Bonde JP, Jensen C, Sumbundu A, Skovbjerg S. Mindfulnessbased cognitive therapy (MBCT) for multiple chemical sensitivity (MCS): Results from a randomized controlled trial with 1year follow-up. J Psychosom Res. 2015 Jul 16. pii: S00223999(15)00487-0.

${ }^{15}$ Fernández-Solà J, Nogué Xarau S. Sensibilidad Química y Ambiental Múltiple. Sobrevivir en un entorno tóxico. Barcelona: Viena Ediciones, 2011.

${ }^{16}$ Lee JH, Jeong I, Kim I. Prevalence and related factors of multiple chemical sensitivity in patients having allergic diseases [Abstract]. Journal of Allergy and Clinical Immunology. 2013;131(2):AB163.

${ }^{17}$ Nogué S, Duenas A, Ferrer A, Fernández J. Sensibilidad química múltiple. Med Clin (Barc). 2011;136(15):683-7.

${ }^{18}$ Caccamo D, Cesareo E, Mariani S, Raskovic D, Ientile R, Curro M, et al. Xenobiotic sensor- and metabolism-related gene variants in environmental sensitivity-related illnesses: a survey on the Italian population. Oxid Med Cell Longev. 2013;2013:831969.

${ }^{19}$ Holst H, Arendt-Nielsen L, Mosbech H, Serup J, Elberling J. Capsaicin-induced neurogenic inflammation in the skin in patients with symptoms induced by odorous chemicals. Skin Res Technol. 2011;17(1):82-90.

${ }^{20}$ Holst H, Arendt-Nielsen L, Mosbech H, Elberling J. Increased capsaicin-induced secondary hyperalgesia in patients with multiple chemical sensitivity. Clin J Pain. 2011;27(2):156-62.

${ }^{21}$ Berg ND, Linneberg A, Thyssen JP, Dirksen A, Elberling J. Non-allergic cutaneous reactions in airborne chemical sensitivity--a population based study. Int $\mathbf{J}$ Hyg Environ Health. 2011;214(3):239-45.

${ }^{22}$ Katerndahl DA, Bell IR, Palmer RF, Miller CS. Chemical intolerance in primary care settings: prevalence, comorbidity, and outcomes. Ann Fam Med. 2012;10(4):357-65.

${ }^{23}$ Skovbjerg S, Rasmussen A, Zachariae R, Schmidt L, Lund R, Elberling J. The association between idiopathic environmental intolerance and psychological distress, and the influence of social support and recent major life events. Environ Health Prev Med. 2012;17(1):2-9.

${ }^{24}$ Skovbjerg S, Christensen KB, Ebstrup JF, Linneberg A, Zachariae R, Elberling J. Negative affect is associated with development and persistence of chemical intolerance: a prospective populationbased study. J Psychosom Res. 2015 May;78(5):509-14.

${ }^{25}$ Nordin S, Ljungberg JK, Claeson AS, Neely G. Stress and odor sensitivity in persons with noise sensitivity. Noise Health. 2013;15(64):173-7.

${ }^{26}$ Skovbjerg S, Berg ND, Elberling J, Christensen KB. Evaluation of the quick environmental exposure and sensitivity inventory in a Danish population. J Environ Public Health. 2012;2012:304314.

27 Barnig C, De Blay F. Pathophysiology of multiple chemical sensitivity. Rev Mal Respir. 2013;30(6):446-50.

${ }^{28}$ Martini A, Iavicoli S, Corso L. Multiple chemical sensitivity and the workplace: current position and need for an occupational health surveillance protocol. Oxid Med Cell Longev. 2013;2013:351457.

${ }^{29}$ Mena G, Sequera VG, Nogue-Xarau S, Rios J, Bertran MJ, Trilla A. [Translation and cross-cultural adaptation of the Quick Environmental Exposure and Sensitivity Inventory for use in the Spanish 
population]. Med Clin (Barc ). 2013;140(7):302-4.

${ }^{30}$ Gibson PR, Sledd LG, McEnroe WH, Vos AP. Isolation and lack of access in multiple chemical sensitivity: A qualitative study. Nurs Health Sci. 2011;13(3):232-7.

${ }^{31}$ Soderholm A, Soderberg A, Nordin S. The experience of living with sensory hyperreactivityaccessibility, financial security, and social relationships. Health Care Women Int. 2011;32(8):686-707.

${ }^{32}$ Dupas D, Dagorne MA. [Multiple chemical sensitivity: a diagnosis not to be missed]. Rev Mal Respir. 2013;30(2):99-104.

${ }^{33}$ Genuis SJ. Chemical sensitivity: pathophysiology or pathopsychology? Clin Ther. 2013;35(5):5727.

34 Ralph B, Martine O, Jacques R. Double-blind non-controlled chemical challenge with environmental toxicological assessment in a Multiple Chemical Sensitivity case. J Neurol Sci. 2011;306(1-2):154-6.

35 Waddick CJ. Healthy residential developments: Reducing pollutant exposures for vulnerable populations with multiple chemical sensitivities [Abstract]. Dissertation Abstracts International Section A: Humanities and Social Sciences. 2011;72(6-A).

${ }^{36}$ Mischley LK, Vespignani MF, Finnell JS. Safety survey of intranasal glutathione. J Altern Complement Med. 2013;19(5):459-63.

${ }^{37}$ Bolt HM, Kiesswetter E. Is multiple chemical sensitivity a clinically defined entity? Toxicol Lett. 2002 Mar 10;128(1-3):99-106.

38 Cullen MR. The worker with multiple chemical sensitivities: an overview. Occup Med 1987;2(4):655-61)

${ }^{39}$ Dantoft TM, Andersson L, Nordin S, Skovbjerg S. Chemical intolerance. Curr Rheumatol Rev. 2015;11(2):167-8.

${ }^{40}$ Mizukoshi A, Kumagai K, Yamamoto N, Noguchi M, Yoshiuchi K, Kumano H, Sakabe K, Yanagisawa Y. In-situ Real-Time Monitoring of Volatile Organic Compound Exposure and Heart Rate Variability for Patients with Multiple Chemical Sensitivity. Int J Environ Res Public Health. 2015 Oct 5;12(10):12446-65.

${ }^{41}$ Rodgers K. Idiopathic environmental intolerances. Reference Module in biomedical Sciences, from Encyclopedia of Toxicology (Third edition), 2014, Pages 996-999, Current as of 1 September 2014.

${ }^{42}$ Andersson L, Claeson AS, Dantoft TM, Skovbjerg S, Lind N, Nordin S. Chemosensory perception, symptoms and autonomic responses during chemical exposure in multiple chemical sensitivity. Int Arch Occup Environ Health. 2015 Apr 28.

${ }^{43}$ Dantoft TM, Elberling J, Brix S, Szecsi PB, Vesterhauge S, Skovbjerg S. An elevated proinflammatory cytokine profile in multiple chemical sensitivity. Psychoneuroendocrinology, 2014, 40, 140-50.

${ }^{44}$ Winder C. Mechanisms of multiple chemical sensitivity. Toxicol Lett. 2002 Mar 10;128(1-3):85-97.

${ }^{45}$ Fujimaki H. Yamamoto S. Tin-Tin-Win-Shwe Hojo R. Sato F. Kunugita N. Arashidani K. Effect of long-term exposure to low-level toluene on airway inflammatory response in mice. Toxicol Lett. 2007 168(2): 132-9.

${ }^{46}$ Alessandrini M, Micarelli A, Chiaravalloti A, Bruno E, Danieli R, Pierantozzi M, Genovesi G, Öberg J, Pagani M, Schillaci O. Involvement of Subcortical Brain Structures During Olfactory Stimulation in Multiple Chemical Sensitivity. Brain Topogr. 2016 Mar;29(2):243-52.

${ }^{47}$ Sage C. The implications of non-linear biological oscillations on human electrophysiology for electrohypersensitivity (EHS) and multiple chemical sensitivity (MCS). Rev Environ Health. 2015 Dec 1;30(4):293-303.

${ }^{48}$ Chiaravalloti A, Pagani M, Micarelli A, Di Pietro B, Genovesi G, Alessandrini M, Schillaci O. Cortical activity during olfactory stimulation in multiple chemical sensitivity: a (18)F-FDG PET/CT study. Eur J Nucl Med Mol Imaging. 2015 Apr;42(5):733-40. 
${ }^{49}$ Azuma K, Uchiyama I, Tanigawa M, Bamba I, Azuma M, Takano H, Yoshikawa T, Sakabe K. Assessment of cerebral blood flow in patients with multiple chemical sensitivity using nearinfrared spectroscopy--recovery after olfactory stimulation: a case-control study. Environ Health Prev Med. 2015 May;20(3):185-94.

${ }^{50}$ Nadeau G, Lippel K. From individual coping strategies to illness codification: the reflection of gender in social science research on multiple chemical sensitivities (MCS). Int J Equity Health. 2014 Sep 10;13:78.

${ }^{51}$ Lohiya GS, Lohiya S, Lohiya S, Krishna V. Scombrotoxinism: Protracted Illness following Misdiagnosis in the Emergency Department. Case Rep Emerg Med. 2015;2015:597934.

${ }^{52}$ Baliatsas C, van Kamp I, Hooiveld M, Lebret E, Yzermans J. The relationship of modern health worries to non-specific physical symptoms and perceived environmental sensitivity: A study combining self-reported and general practice data. J Psychosom Res. 2015 Nov;79(5):355-61.

53 David Suzuki Foundation. The "dirty dozen" ingredients investigated in the David Suzuki Foundation Survey of Chemicals in Cosmetics. October 2010.

${ }^{54}$ Gundersen H, Harris A, Bråtveit M, Moen BE. Odor-related Chronic Somatic Symptoms Are Associated with Self-Reported Asthma and Hay Fever: The Hordaland Health Study. Iran J Allergy Asthma Immunol. 2015 Feb;14(1):19-27

${ }^{55}$ Ross PM, Whysner J, Covello VT, Kuschner M, Rifkind AB, Sedler MJ, Trichopoulos D, Williams GM. Olfaction and symptoms in the multiple chemical sensitivities syndrome. Prev Med. 1999 May;28(5):467-80.

${ }^{56}$ Ec.europa.eu [Internet]. EU: European Union. 2014 [updated 24 Ago 2015; cited 4 nov 2015]. Available at: http://ec.europa.eu/consumers/sectors/cosmetics/files/pdf/sue_reporting_guidelines _en.pdf

${ }^{57}$ Fukuyama T. Ueda H. Hayashi K. Tajima Y. Shuto Y. Saito T.R. Harada T. Kosaka T. Detection of low-level environmental chemical allergy by a long-term sensitization method. Toxicol Lett. 2008 180:(1): 1-8.

${ }^{58}$ Bensefa-Colas L; Dupas D. Idiopathic environmental intolerance: 2 disabling entities to recognize. Rev Prat. 2014, Mar; 64(3):358-62.

${ }^{59}$ Alobid I, Nogué S, Izquierdo-Dominguez A, Centellas S, Bernal-Sprekelsen M, Mullol J. Multiple chemical sensitivity worsens quality of life and cognitive and sensorial features of sense of smell. Eur Arch Otorhinolaryngol. 2014 Dec;271(12):3203-8.

${ }^{60}$ Cui X, Lu X, Hiura M, Oda M, Hisada A, Miyazaki W, Omori H, Katoh T. Prevalence and interannual changes in multiple chemical sensitivity in Japanese workers. Environ Health Prev Med. 2014 May;19(3):215-9.

${ }^{61}$ Vuokko A, Selinheimo S, Sainio M, Suojalehto H, Järnefelt H, Virtanen M, Kallio E, Hublin C, Karvala K. Decreased work ability associated to indoor air problems - An intervention (RCT) to promote health behavior. Neurotoxicology. 2015 Jul;49:59-67.

${ }^{62}$ Van Dongen D; Smid T; Timmermans DR. Symptom attribution and risk perception in individuals with idiopathic environmental intolerance to electromagnetic fields and in the general population. Perspect Public Health. 2014, May; 134(3):160-8.

\section{Annex 1. Search strategies}

MEDLINE

Dec 2015

\#1 "Multiple Chemical Sensitivity"[Mesh]

\#2 Multiple[tiab] AND (Chemical Sensitivit*[tiab] OR chemical hypersensit*[tiab])

\#3 Multiple[ti] AND chemica*[ti] AND (sensitivit*[ti] OR hypersensitivit*[ti])

\#4 Idiopathic Environmental Intoleranc*[tiab]

\#5 (\#1 OR \#2 OR \#3 OR \#4) 
("2011/01/01"[PDat] : "2015/12/15"[PDat])

Epidemiology

incidenc* OR prevalenc* OR epidemiol*

Clinical manifestations and comorbidity

symptoms* OR signs* OR manifestat* OR comorbidit*

Diagnostic

diagnos* OR prognos* OR screen* OR specific*

Quality of life

quality* OR lif* OR impact* OR psychol*

Treatment

therap* OR trial*

Prevention

prevent* OR control*

EMBASE

Dec 2015

\#1 "Multiple Chemical Sensitivity"/exp

\#2 Multiple:ab AND (“Chemical Sensitivity”:ab OR “chemical hypersensitivity”:ab)

\#3 Multiple:ti AND chemica*:ti AND (sensitivit*:ti OR hypersensitivit*:ti)

\#4 "Idiopathic Environmental Intolerance":ab,ti

\#5 (\#1 OR \#2 OR \#3 OR \#4)

Epidemiology

incidenc* OR prevalenc* OR epidemiol* AND [embase]/lim AND [2010-2015]/py

Comorbilidity

symptoms* or signs* or manifestat* or comorbidit* AND [embase]/lim AND [2010-2015]/py

Diagnostic

diagnos* OR prognos* OR screen* OR specific* AND [embase]/lim AND [2010-2015]/py

Quality of life

quality or lif* or impact* or psychol* AND [embase]/lim AND [2010-2015]/py

Treatment

therap* or trial* AND [embase]/lim AND [2010-2015]/py

Prevention

prevent* or control* AND [embase]/lim AND [2010-2015]/py

\section{PUBPSYCH}

Dec 2015

\#1 multiple chemical sensitivity

\#2 idiopathic environmental intolerance

\#1 OR \#2

Dates: 2010-2015 


\section{PSYCINFO}

Dec 2015

S1 - AB "chemicalsensitivit*"

S2 - AB "chemical hypersensit*"

S3 - AB "multiple"

S4 - S1 OR S2

S5 - S3 AND S4

S6 - TI multiple

S7 - TI "chemica*"

S8 - TI "sensitivit*"

S9 - TI "hypersensitivit*"

S10 - S8 OR S9

S11 - S6 AND S7 AND S10

S12 - TI "idiopathicenvironmentalintoleranc*"

S13 - AB "idiopathic environmental intoleranc*"

S14 - S12 OR S13

S15 - TI "multiple chemical sensitivity"

S16 - AB "multiple chemical sensitivity"

S18 - S15 OR S16

S19 - S5 OR S11 OR S14 OR S17, Limiters - Publication Year: 2010-2015

\section{The COCHRANE LIBRARY}

Dec 2015

\#1 MeSH descriptor: [Multiple Chemical Sensitivity] explode all trees

\#2 idiopathic environmental intoleranc* (Word variations have been searched)

\#3 multiple chemical intolerance (Word variations have been searched)

\#1 or \#2 or \#3

\section{Treatment}

therap* or trial*:ti,ab,kw (Word variations have been searched)

\section{CRD:}

Dec 2015

\#1 (multiple AND chemical AND sensitivity)

\#2 (idiopathic AND environmental AND intolerance)

\#3 (multiple AND chemical AND hypersensitivity)

\#4 \#1 OR \#2 OR \#3 FROM 2010 TO 2015

\section{TOXLINE:}

Dec 2015

\# 1 "idiopathic environmental intolerance" AND 2010:2015 [yr] [not] PubMed [org] [not] pubdart [org] 
Alberto Frutos Pérez-Surio et al.

\# 2 "multiple chemical sensitivity" AND 2010:2015 [yr] [not] PubMed [org] [not] pubdart [org]

\section{CISDOC database}

Dec 2015

\#1 multiple chemical sensitivity

\#2 idiopathic environmental intolerance 